\title{
Interfaces entre a educação permanente e a educação interprofissional em saúde
}

\author{
Interfaces between permanent education and interprofessional education in health \\ Interfaces entre la educación permanente y la educación interprofesional en salud
}

Como citar este artigo:

Ogata MN, Silva JAM, Peduzzi M, Costa MV, Fortuna CM, Feliciano AB. Interfaces between permanent education and interprofessional education in health. Rev Esc Enferm USP. 2021;55:e03733. https://doi.org/10.1590/S1980-220X2020018903733

\author{
Márcia Niituma Ogata ${ }^{1}$ \\ Jaqueline Alcantara Marcelino da \\ Silva ${ }^{1}$ \\ Marina Peduzzi ${ }^{2}$ \\ Marcelo Viana Costa ${ }^{3}$ \\ Cinira Magali Fortuna ${ }^{4}$ \\ Adriana Barbieri Feliciano ${ }^{1}$ \\ ${ }^{1}$ Universidade Federal de São Carlos, \\ Departamento de Enfermagem, \\ São Carlos, SP, Brasil. \\ ${ }^{2}$ Universidade de São Paulo, Escola de \\ Enfermagem, Departamento de Orientação \\ Profissional, São Paulo, SP, Brasil. \\ ${ }^{3}$ Universidade Federal do Rio Grande do \\ Norte, Escola Multicampi de Ciências \\ Médicas, Caicó, RN, Brasil. \\ ${ }^{4}$ Universidade de São Paulo, Escola \\ de Enfermagem de Ribeirão Preto, \\ Departamento Materno-Infantil e Saúde \\ Pública, Ribeirão Preto, SP, Brasil.
}

\begin{abstract}
This was a reflective theoretical essay that was based on the national and international literature. The goal was to analyze the interfaces and historical-conceptual distinctions between permanent education in health and interprofessional education in health. In the international context, there have been educational movements aimed at health workers with an emphasis on the incentives of the Pan-American Health Organization. In Brazil, both proposals highlight a commitment to the quality of practice in the Unified Health System, focused on health needs, with approaches that have a lot in common. Permanent education in health is geared toward on-the-job training to transform work processes, with the objective of delivering comprehensive care. Interprofessional education in health involves shared interactive learning of collaborative skills for effective teamwork, guided by interprofessional collaboration. The purpose of both is to qualify health practices through the education of workers in groups; however it is essential to distinguish the theoretical, conceptual, and methodological frameworks that support them.
\end{abstract}

\section{DESCRIPTORS}

Health Education; Work Engagement; Interprofessional Relations; Interprofessional Education.
Autor correspondente:

Márcia Niituma Ogata

Rua Lilia Elisa Eberle Lupo, 151,

casa 91, Jardim Salto Grande

CEP 14803-886 - Araraquara, SP, Brasil

ogata@ufscar.br 


\section{INTRODUÇÃO}

O Sistema Único de Saúde (SUS) requer profissionais comprometidos com os princípios da integralidade, equidade e universalidade. O processo de fortalecimento do SUS é marcado por políticas de reorientação da formação e do trabalho em saúde que historicamente buscam enfrentar gargalos que comprometem a operacionalização do sistema segundo seus princípios. Nesse movimento histórico, destacam-se dois grandes temas: a Educação Permanente em Saúde (EPS) e a Educação Interprofissional em Saúde (EIP). A primeira constitui a política do SUS para a formação dos trabalhadores do setor ${ }^{(1)}$, e a segunda, uma abordagem de educação e formação profissional em saúde presente no cenário internacional desde os anos $1970^{(2)}$, que se amplia no país na última década.

Os movimentos globais de mudança na lógica do trabalho e da formação em saúde têm centrado esforços no fortalecimento dos sistemas de saúde, especialmente na mudança do modelo de atenção hegemônico, centrado na doença e/ou nos profissionais, para um modelo voltado para as necessidades das pessoas, famílias, comunidades e do território. A EPS e a EIP são reconhecidas como referenciais teórico-conceituais e metodológicos importantes ${ }^{(3-5)}$ e podem ser complementares para o fomento de práticas que proporcionem a mudança esperada para implementação da integralidade do cuidado, acesso universal e qualidade da atenção à saúde.

Apesar da relevância da EPS no processo de construção e fortalecimento do SUS, cabe destacar sua polissemia, na medida em que muitas são as práticas denominadas de EPS, sendo que algumas são mais próximas da educação continuada (EC).

Considera-se a especificidade de cada termo em função do aspecto mais amplo da EPS e da $\mathrm{EC}^{(5)}$, bem como da inserção, para alguns autores, de uma na outra como práticas complementares ${ }^{(6)}$. Outros termos são utilizados como sinônimos, incluindo a aprendizagem ao longo da vida - terminologia mais comumente utilizada na comunidade europeia ${ }^{(3-6)}$. A Política Nacional de EPS ${ }^{(7)}$ e algumas produções de autores de referência na área ${ }^{(3-4)}$ estabelecem distinções entre EPS e EC, considerando o pressuposto educacional que as difere quanto: ao objetivo principal (transformação de práticas e aprendizagem significativa ou como transmissão de conhecimento), ao público a que se destinam (foco na interdisciplinaridade e multiprofissionalidade ou nas categorias profissionais), à forma como se organizam (na valorização do trabalho como fonte de conhecimento ou no conhecimento técnico científico de cada área) e às estratégias pedagógicas (estratégias de ensino participativas que propiciam a reflexão das práticas ou com ênfase em cursos e treinamentos pontuais ${ }^{(5)}$.

No movimento histórico de permanente reflexão sobre os desafios relacionados à formação dos profissionais de saúde, o debate sobre EIP vem ganhando importante visibilidade - em todo o mundo e no Brasil - demandando uma discussão mais cuidadosa sobre suas imprecisões e confusões conceituais. A literatura aponta a EIP como abordagem que busca promover a articulação entre as profissões da saúde com base na colaboração( ${ }^{(8)}$, que ocorre quando profissionais de diferentes áreas trabalham juntos, porque reconhecem essa dinâmica como capaz de produzir melhores resultados na atenção à saúde de usuários, famílias e comunidade do território ${ }^{(5)}$. Apesar do crescente interesse pela temática da EIP no Brasil, ainda há a necessidade de se elaborar evidências científicas sobre os movimentos precursores dessa discussão. A ampliação do debate sobre EIP no país acompanha o movimento global de valorização da interprofissionalidade na formação como proposta de enfrentamento da histórica fragmentação do trabalho em saúde e educação uniprofissional.

A EPS e a EIP consistem em movimentos que enfrentam desafios inerentes a todo processo de (des)construção dos modos de pensar a educação das profissões da saúde no contexto brasileiro. Nesse sentido, dependendo de como são operadas, estão comprometidas com uma perspectiva democrática da construção do conhecimento, na contramão da lógica mercantilista que se coloca para a saúde, considerando seu alinhamento com os princípios democráticos do sistema de saúde brasileiro e o engajamento pela manutenção dos direitos sociais e do acesso à saúde pela população.

As contínuas aproximações dos autores na construção dos campos teóricos da EPS e da EIP, em cenários estratégicos da formação e do trabalho em saúde, tais como Comissão de Integração Ensino-Serviço, Núcleo de EPS, Programa de Educação para o Trabalho em Saúde (PETSaúde/PET-Saúde Interprofissionalidade), Programa Nacional de Reorientação da Formação Profissional em Saúde (Pró-Saúde), Residência Multiprofissional, Mestrados Profissionais, bem como iniciativas de desenvolvimento docente, atenção à saúde nos serviços, ensino prático nos diferentes cenários do sistema de saúde e projetos de pesquisa regionais e nacionais, evidenciaram os desafios relacionados à compreensão do conceito de EPS e EIP por parte dos diferentes atores (gestores, trabalhadores, docentes, estudantes e usuários).

Nesse contexto, a elaboração do presente ensaio teórico de cunho reflexivo foi conduzida pela seguinte questão: quais as interfaces e as diferenças entre os pressupostos teórico-conceituais e metodológicos da EIP e a EPS e como elas dialogam com o movimento de reorientação da formação e do trabalho em saúde no contexto do SUS? Tomando por base a contextualização da literatura nacional e internacional e o questionamento realizado, o artigo tem o objetivo de analisar as interfaces e distinções histórico-conceituais entre EPS e EIP.

\section{CONTEXTO DE ORIGEM E IMPLEMENTAÇÃO DOS CONCEITOS DE EPS E EIP}

$\mathrm{O}$ termo Educação Permanente (EP) tem sua origem na área de educação, sendo a França seu principal cenário (embora não seja o primeiro), numa perspectiva de tratar do prolongamento da escolaridade obrigatória e da reforma do ensino público ${ }^{(2)}$. No final da década de 1960 , a Organização das Nações Unidas para a Educação, a Ciência e a Cultura (Unesco) incorporou a ideia de qualificação do capital 
humano, considerando-o como um dos aspectos mais importantes para a produtividade econômica e o desenvolvimento de um país. Num contexto do neocapitalismo, o processo de automação do trabalho, com a substituição das pessoas pelas máquinas, provocou consequências para o trabalho humano e para sua qualificação ${ }^{(2)}$. A EP foi inicialmente relacionada às necessidades de conhecimentos específicos no processo de acumulação do capitalismo tardio ${ }^{(2)}$ e pode ser compreendida como uma ferramenta ideológica do Estado, impondo aos trabalhadores a ideia de que existem novas formas de trabalho centradas nas necessidades do modo de produção econômica e política ${ }^{(2)}$. A obra $A$ educação contra a educação: o esquecimento da educação e a educação permanente ${ }^{(2)}$ apresenta um extenso estudo analítico da EP nos aspectos fenomenológico, hermenêutico e filosófico, com importante contribuição na reflexão da EP. Apesar de trazer, no seu bojo, a ideia de que a educação deve continuar durante toda a vida, articula a intencionalidade de formar para enfrentar as mudanças do mundo do trabalho dominadas pelas técnicas e pelas ciências. Contudo, desconsidera o enfrentamento das desigualdades sociais reforçando-as na perspectiva meritocrática.

Em meados da década de 1980, a Organização PanAmericana de Saúde (OPAS) se apropriou do termo EP, tendo em vista a renovação do debate sobre as ações educacionais voltadas a trabalhadores de saúde e o entendimento de que a formação é responsabilidade dos sistemas de saúde e busca promover mudanças das práticas dos profissionais ${ }^{(9)}$. O estudo analisa a produção da OPAS sobre a educação dos profissionais de saúde no período de 1975 e 2002 e resulta no reconhecimento de duas matrizes conceituais: a primeira, de 1975 a 1984, denominada EC e a segunda, de 1984 a 2002, EP. A primeira matriz conceitual constitui-se com base nos seguintes componentes: pedagogia da transmissão, momentos educacionais delimitados, identificação de necessidades e objetivos por quem está fora do contexto, determinação centralizada de prioridades, participação regulada do conjunto de pessoal da saúde. Esta última definida como a soma de ações passíveis de serem realizadas isoladamente, no âmbito do saber e fazer de cada profissão ${ }^{(9)}$.

A segunda matriz é construída pelos componentes: educação no trabalho, pelo trabalho e para o trabalho, pedagogia da problematização, participação ampliada, enfoque estratégico e interprofissionalidade ${ }^{(9)}$. Segundo os autores, essa matriz conceitual reconhece o trabalho como potência educacional e de produção do conhecimento na formação e no desenvolvimento de trabalhadores de saúde ${ }^{(9)}$ aproximando-se dos pressupostos previstos na constituição do SUS. No Brasil, houve o acréscimo de "em saúde" ao termo EP indicando tratar-se de uma aposta de estratégia para uma recomposição das práticas de cuidado, gestão, formação e participação da sociedade no SUS. Na origem da EPS, observa-se que ela ocupa um papel contra-hegemônico de organização do processo de trabalho e de cuidado que permite refletir sobre os desafios que ela se coloca na (des)construção de formas reificadas de aprender, gerir e formar em saúde.

Em atendimento à Constituição Federativa de 1988, foi criada, no Ministério da Saúde, em 2003, a Secretaria de Gestão do Trabalho e da Educação na Saúde (SGTES), que tinha como responsabilidade a formulação de políticas orientadoras da gestão, formação, educação e regulação dos trabalhadores de saúde. Inicialmente, essa secretaria foi composta pelo Departamento de Gestão da Regulação do Trabalho em Saúde (DEGERTS) e o Departamento de Gestão da Educação na Saúde (Deges), este responsável pela implantação da Política Nacional de EPS (PNEPS) instituída em 2004 ${ }^{(7)}$.

A PNEPS constitui-se numa política pública estratégica para fomentar mudanças nos processos de formação e desenvolvimento dos trabalhadores da saúde no SUS, tendo como pressupostos a problematização da realidade do cotidiano de trabalho para a reflexão e construção coletiva de solução dos problemas permitindo o delineamento de novos conceitos e paradigmas, bem como a oportunidade de disparar mudanças efetivas nos processos de trabalho ${ }^{(7)}$. Desse modo, desde 2004, a EPS constitui o conceito norteador de uma política transversal de educação e formação dos trabalhadores do SUS. Essa política vem atender ao preceito constitucional do Art. 200, inciso III ${ }^{(10)}$, que prevê para o SUS a responsabilidade constitucional, dentre outras, de ordenar a formação de trabalhadores na área da saúde e tem como princípio norteador a aprendizagem ancorada no processo de trabalho em saúde e a integração com órgãos de formação.

Uma estratégia implementada a partir da Portaria $n^{\circ}$ $198^{(7)}$ foi a criação de colegiados na forma de Polos de EPS, que tinham como objetivo efetivar a condução da política locorregional. Os Polos consistiam em dispositivos do SUS para promover mudanças nas práticas da atenção e da educação na saúde, funcionando como rodas de debate e de construção coletiva - Rodas para a EPS - com a participação de representantes dos níveis de gestão, trabalhadores, instituições formadoras (docentes e estudantes) e usuários. Isso propiciou a aproximação dos diferentes atores para pensar, debater e formular mudanças na formação em saúde.

A partir de 2007, essa política sofreu alterações considerando as mudanças no sistema de gestão da saúde, com o intuito de fortalecer a gestão local e o controle social ${ }^{(11)}$. Mudanças institucionais ocorreram na gestão federal, após a implantação da política de EPS, que repercutiram em ressignificações conceituais e metodológicas na implementação da EPS, ocasionando alterações, como a substituição dos Polos pelas Comissões de Integração Ensino-Serviço (CIES) e a participação dos Colegiados de Gestão Regional na gestão da EPS. Essa substituição provocou uma alteração sensível no processo que havia sido disparado inicialmente pelos Polos de EPS, fundamentalmente na perspectiva da participação dos diferentes atores na construção coletiva dos projetos locais de EPS. Estudo sobre as CIES no Brasil ${ }^{(12)}$ aponta sua existência em diversos Estados e as dificuldades relacionadas aos modos de operar e articular ações de EPS nos diferentes territórios.

A PNEPS tem foco no processo de trabalho e no desenvolvimento dos profissionais por meio da permanente articulação ensino-serviço ${ }^{(7,10)}$, visando não somente à formação mas também à qualificação da gestão e do processo de trabalho em saúde. Uma análise das ações do Ministério da Saúde em 2017 e 2018, para a retomada do processo de implementação 
da PNEPS, relata que, nas oficinas para a discussão do tema, observou-se falta de compreensão de gestores sobre a EPS e o modo de funcionamento das CIES, baixa articulação do quadrilátero, financiamento insuficiente, modelo de formação ainda tradicional com reprodução de cursos e baixa apropriação da concepção a respeito da $\mathrm{EPS}^{(12)}$. O que o estudo não apresenta e que não pode ser desconsiderado são as mudanças ocorridas no cenário político nacional que interferem diretamente na implementação da PNEPS segundo seus pressupostos fundantes. Destaca-se, por exemplo, a aprovação da Emenda Constitucional $\mathrm{n}^{\circ} 95$, que congela os gastos públicos por 20 anos e piora o cenário de desfinanciamento do SUS, assim como as mudanças da Política Nacional de Atenção Básica ${ }^{(13)}$ que atestam a falta de compromisso com políticas públicas de fortalecimento do SUS. A implementação de políticas públicas se dá em situação de embates e disputas que não são técnicas, mas são, sobretudo, políticas no sentido da intencionalidade e apostas alicerçadas em valores e posições dos agentes que as operam nos diversos planos de ação.

De modo semelhante à EP, a origem oficial da EIP pode ser atribuída a um grupo de experts da Organização Mundial de Saúde (OMS) que, em 1973, defendeu a incorporação da formação interprofissional como resposta à necessidade do trabalho em equipe e da abordagem integral das necessidades de saúde dos usuários dos sistemas de saúde ${ }^{(2)}$. Posteriormente, no encontro de Alma Ata de 1978, a EIP foi destacada como uma estratégia necessária no relatório "Saúde para todos no ano 2000"(14).

Em 1988, a OMS publicou o relatório Learning together to work together for healt ${ }^{(15)}$, no qual declarou o compromisso com um modelo de formação voltado às necessidades de saúde da população, com o desenvolvimento do aprendizado interativo com outras áreas profissionais e de competências para o trabalho em equipe. Contudo, na época, o termo interprofissional ainda era mencionado, na literatura, como sinônimo de multiprofissional, sendo este último definido pela OMS como o aprendizado de estudantes ou trabalhadores de forma conjunta e interativa, num período determinado, para construir ações de promoção, prevenção e recuperação de forma colaborativa ${ }^{(15)}$.

O estímulo da OMS à EIP culminou com a publicação do "Marco para a Ação em Educação Interprofissional e Prática Colaborativa" em 2010. No mesmo ano, o grupo The Lancet Commission Health Professional for a New Century publicou um artigo que enfatizou a EIP como uma resposta ao aumento da complexidade das necessidades de saúde dos usuários dos sistemas de saúde, considerando o envelhecimento populacional, o aumento das doenças crônicas e da incorporação tecnológica. Defendeu a integração entre o sistema educacional e o sistema de saúde, de modo que a formação e a saúde se retroalimentassem em suas demandas. Os autores destacaram que o hegemônico modelo Flexner da formação em saúde precisava ser superado com a incorporação da interprofissionalidade na formação das diversas profissões da saúde, de métodos ativos de ensino-aprendizagem que preconizassem o protagonismo dos estudantes, de reformas curriculares que privilegiassem a EIP e o compromisso com a formação para o trabalho em equipe interprofissional $^{(16-17)}$. A hegemonia desta matriz flexneriana, presente na maioria das instituições de ensino no Brasil, pode ser considerada um dos nós críticos no processo de implantação da EPS e da EIP. Posteriormente, o relatório intitulado "Estratégia Global de Recursos Humanos para a Saúde: força de trabalho 2030" reafirmou a indicação da EIP como um modelo necessário de resposta aos sistemas de saúde, preparo para o trabalho em equipes interprofissionais e colaboração ${ }^{(18)}$.

Historicamente, o movimento da EIP no Reino Unido iniciou-se por volta de 1966, seguido pelo Canadá e Estados Unidos, assim como por outros países europeus, Austrália e, mais recentemente, Ásia e África. Por mais de quatro décadas, esforços têm sido realizados para avançar em direção à colaboração interprofissional centrada no usuário, por ser reconhecida como uma estratégia para a resolução de problemas dos sistemas de saúde ${ }^{(19-20)}$.

Em 1969, no Canadá, foi divulgada a primeira publicação sobre a temática da EIP pela Universidade British Columbia e, somente em 2003, mediante um acordo com ministros da saúde, foi criado um Comitê Nacional de Experts para Educação Interprofissional e Prática Colaborativa Centrada no Paciente (IECPCP), que desenvolveu diversas pesquisas e publicações. Essa iniciativa buscou aproximar a formação dos serviços de saúde, cuja articulação é indispensável para o fortalecimento da EIP e da colaboração interprofissional, reconhecidas como práticas necessárias para a sustentabilidade do sistema de saúde, as quais têm sido veiculadas pela Canadian Interprofessional Health Collaborative (CIHC) ${ }^{(19-20)}$.

No Reino Unido, destaca-se o Center for the Advancement of Interprofessional Education (Caipe), criado em 1987, com o intuito de promover colaboração entre profissionais de diferentes áreas por meio da EIP, voltada para a atenção integral e efetiva das necessidades de saúde dos usuários. Nesse sentido, as ações do Caipe foram projetadas para coordenar iniciativas de EIP, promover a disseminação de informações, experiências e pesquisas, desenvolver oportunidades para compartilhar o aprendizado e fortalecer a perspectiva interprofissional na formação profissional ${ }^{(21)}$.

No Brasil, movimentos mais conhecidos, e de longa data, relacionados à reorientação da formação dos profissionais de saúde incorporaram a interdisciplinaridade como um dos princípios formativos, marcadamente nas iniciativas de reforma curricular, na perspectiva do currículo integrado e/ ou encorajados pelas diretrizes curriculares nacionais para os cursos de graduação em saúde, especialmente nos currículos baseados em competências, nos quais o trabalho em equipe ganha maior importância.

O marco principal da incorporação da EIP no Brasil foi a criação, em 2006, da proposta interprofissional do Campus Baixada Santista da Universidade Federal de São Paulo (Unifesp) $^{(22)}$. No mesmo ano, foi criada a Universidade Federal do Recôncavo da Bahia com a proposta do Bacharelado Interdisciplinar em Saúde (BIS), que também foi adotada na Universidade Federal da Bahia, com apoio do Programa de Reestruturação e Expansão das Universidades Federais Brasileiras (Reuni) do Ministério da Educação $(\mathrm{MEC})^{(23)}$. Em 2008, iniciou-se o projeto interdisciplinar 
da Universidade de Brasília (UnB), Campus Ceilândia, e, em 2013, na Universidade Federal do Sul da Bahia ${ }^{(24)}$, foi adotada a proposta do BIS com ênfase no novo modelo de formação por ciclos. As experiências das universidades mencionadas são exitosas na incorporação de metodologias e de pressupostos teórico-conceituais potentes no processo de formação de profissionais mais aptos ao efetivo trabalho em equipe, por meio do desenvolvimento intencional e sistematizado de competências colaborativas ${ }^{(22-24)}$. Cabe destacar que o currículo integrado dos cursos de graduação da Unifesp Baixada Santista está fundamentado na EIP e os demais, na interdisciplinaridade articulada à EIP.

Interdisciplinaridade diz respeito ao domínio do conhecimento científico e das disciplinas, enquanto a interprofissionalidade se refere ao âmbito das práticas profissionais e profissões. A imprecisão dos conceitos e termos relativos às duas abordagens, interprofissional e interdisciplinar, denota a ausência de consenso sobre os respectivos elementos-chave ${ }^{(5)}$. Ressalta-se a complementaridade entre interdisciplinaridade e interprofissionalidade, visto que estudantes e trabalhadores precisam lidar com o desafio de apropriarem-se tanto das mediações entre os conhecimentos das diversas disciplinas quanto das mediações entre as diferentes práticas profissionais presentes na atenção à saúde. Esse debate é relevante à medida que se reconhece a crescente complexidade da saúde que requer enfrentar e superar o reducionismo e a disjunção da racionalidade científica moderna que ocasionou a fragmentação do conhecimento em disciplinas e das práticas de saúde em profissões.

Outro marco histórico, para o movimento da EIP no Brasil, são os Colóquios Internacionais de Educação e Trabalho Interprofissional em Saúde (Cietis) propostos por um grupo de docentes pesquisadores de universidades públicas vinculados a pesquisas, ensino e vivências interprofissionais. $\mathrm{O}$ primeiro Cietis ocorreu em Natal, organizado pela Universidade Federal do Rio Grande do Norte, em 2015, o segundo, em Santos, na Unifesp-Campus Baixada Santista, em 2016, no qual foi criada a Rede Brasileira de Educação e Trabalho Interprofissional em Saúde (ReBETIS), o terceiro Cietis, em Brasília, com organização da Comissão Executiva ReBETIS, em 2017, e o quarto, na UnB Campus Ceilândia, em 2018, com recomposição da referida comissão ${ }^{(24)}$. Todas as edições do evento foram apoiadas pela OPAS e pelo Ministério da Saúde por meio da SGTES. Os Cietis mobilizaram docentes, profissionais, pesquisadores e estudantes da área da saúde a se reunirem anualmente com o intuito de socializar avanços e desafios para o fortalecimento da EIP e da colaboração interprofissional, comprometidos com o desenvolvimento do SUS e a centralidade das necessidades de saúde de usuários, famílias e comunidades na formação e no trabalho.

O mesmo compromisso social com o SUS mobilizou a criação da ReBETIS, com o intuito de disseminar experiências, saberes e práticas que contribuam para a transformação da formação na perspectiva da EIP, preparando para o trabalho em equipe interprofissional e a colaboração em direção à integralidade da atenção em um sistema de saúde de qualidade, mais justo e equânime.
Importantes experiências brasileiras de reorientação da formação profissional fortalecidas pela Política de EPS integraram, na sua construção, o trabalho como princípio educativo. Dentre elas, citam-se Pró-Saúde, PET-Saúde, Residências Multiprofissionais, Ver-SUS, que se aproximam dos princípios/estratégias também valorizados pelo movimento da EIP: comunicação interprofissional, participação dos usuários, das famílias e comunidades, integração ensino-serviço-comunidade, trabalho em equipe, tomada de decisões compartilhadas e colaboração interprofissional. Dessa forma, a EIP no Brasil tem potencial para se articular a esse movimento histórico da EPS.

No âmbito da América Latina e do Caribe, cabe destacar a atuação da OPAS no encorajamento e apoio aos países para a incorporação dos pressupostos da EIP nas políticas orientadoras de mudanças da formação e do trabalho em saúde. $\mathrm{O}$ ano de 2016 foi crucial nesse sentido, tendo em vista a organização pela OPAS e a realização, em Bogotá Colômbia, da I Reunião Técnica de Educação Interprofissional ${ }^{(25)}$. Nessa reunião, foi criada a Rede Regional de Educação Interprofissional das Américas (REIP) como estratégia de articulação e cooperação técnica entre instituições educacionais, organizações profissionais e Ministérios da Saúde e Ministérios da Educação ${ }^{(25)}$, e os países participantes foram chamados a elaborar um Plano de Ação para o Fortalecimento da EIP no respectivo contexto.

O Brasil participou da reunião em Bogotá e, em 2017, realizou a Oficina de Alinhamento Conceitual sobre a Educação e Trabalho Interprofissional tendo o Ministério da Saúde, por meio do Deges/SGTES e a OPAS como propositores, o objetivo de construir um diálogo sobre as bases teórico-conceituais e metodológicas da EIP, mediante parceria entre pesquisadores de instituições de Ensino Superior e a $\operatorname{ReBETIS}{ }^{(26)}$. Na ocasião da referida oficina, foi apresentado e discutido o Plano de Ação de Fortalecimento da EIP no Brasil e disparada a incorporação dessa terminologia na Política Nacional de EPS no SUS.

Como parte do Plano de Ação, a SGTES publicou em 2018 o edital do PET-Saúde Interprofissionalidade, com o objetivo de promover o trabalho como princípio formativo, a integração ensino-serviço-comunidade e a indução de mudanças na formação dos profissionais de saúde a partir da incorporação intencional e sistematizada dos elementos teóricos e metodológicos da EIP nos projetos pedagógicos dos cursos selecionados.

A natureza sócio-histórica dos movimentos da EPS e da EIP sinaliza a busca da construção de uma rede saúde escola que demanda esforços para mudança do modelo hegemônico pautado na reprodução da educação tradicional verticalizada, hierarquizada, com protagonismo centralizado em relações de poder e na perspectiva uniprofissional da formação e do trabalho, que subsidia uma lógica de fragmentação do trabalho e do conhecimento, com importantes implicações no enfrentamento das complexas e dinâmicas necessidades de saúde. Ambos os movimentos educacionais valorizam a emancipação dos sujeitos, a autonomia e a construção coletiva, com a inclusão dos usuários nas mudanças das práticas educacionais e dos processos de trabalho em saúde. 
AS INTERFACES TEÓRICO-CONCEITUAIS E METODOLÓGICAS DA EPS E EIP

No processo de construção do SUS, destacam-se três campos de tensão que podem ser estratégicos na reorientação da educação para o SUS ${ }^{(12)}$ : o território das práticas de saúde, a produção de ações e as organizações.

As práticas de saúde como território de disputas e constituição de políticas do cuidar estão sujeitas a interesses distintos e a capacidades de atuar. A produção de ações de saúde como espaço do trabalho vivo possibilita ao trabalhador atuar na singularidade do contexto de cada usuário apoiado pelas tecnologias leves e utilizando as tecnologias leves-duras e duras de acordo com as necessidades identificadas. Nessa produção viva, há certo grau de incertezas e certo grau de autonomia dos trabalhadores, o que permite possibilidades de criação e inovação. As organizações constituem-se em espaços de intervenção de diferentes atores com capacidade de autogoverno que constroem práticas instituintes e que disputam, no cotidiano do processo de trabalho, com as normas e regras instituídas, mas que podem ser levadas ao interesse do coletivo, em especial, dos usuários ${ }^{(27)}$.

A adoção da EPS e da EIP como referências conceituais para as políticas de reorientação da educação para o SUS deve-se à necessidade de construção de práticas inovadoras de cuidado e de gestão voltadas aos princípios do SUS. Aliado a essa questão, travou-se a crítica de que o formato pedagógico tradicionalmente adotado no ensino em cursos e treinamentos tradicionais, focados na transmissão do conhecimento de natureza estritamente cognitiva, sem articulação entre teoria e cotidiano das práticas, entre ensino e serviço, era pouco potente para a necessidade de mudanças. Nesse sentido, a EPS pressupõe que a educação e formação dos trabalhadores de saúde ocorra numa relação recíproca e dialética com as práticas de atenção à saúde, com potência de transformação dos processos de trabalho, a partir da reflexão do cotidiano vivenciado na gestão, na atenção, no controle social, na problematização dessas vivências e na construção coletiva de novas formas de fazer gestão e cuidado. Para construção de um processo de mudança ou incorporação de novas práticas, é preciso que se identifiquem incômodos, insatisfações ou insuficiências vividas e percebidas como disparadores de mudanças.

O movimento da EIP também expressa o compromisso com a transformação das práticas de saúde no contexto do SUS quando ressalta a ênfase no propósito explícito em promover a colaboração interprofissional e o aprendizado para o efetivo trabalho em equipe ${ }^{(28)}$. Essa ênfase pode ser vista como uma distinção importante entre EPS e EIP. Historicamente a ideia de juntar estudantes ou profissionais de diferentes áreas em um mesmo espaço já se constituía como interprofissional, como se a inserção na dinâmica de trabalho em saúde - considerando o seu caráter eminentemente coletivo - fosse suficiente para estimular a colaboração interprofissional.

A expressão da referida intencionalidade se materializa desde a escolha até a operacionalização dos referenciais teórico-metodológicos da EIP, com levantamento das necessidades educacionais, definição e articulação das competências específicas, comuns e colaborativas, ancoradas em teorias educacionais que promovem o protagonismo dos educandos e metodologias ativas de ensino-aprendizagem que estimulem resultados que não seriam alcançados na perspectiva uniprofissional ${ }^{(29)}$. Essa intencionalidade requer, dessa forma, que as iniciativas educacionais expressem, com clareza, suas contribuições para a formação de profissionais mais aptos à colaboração e ao efetivo trabalho em equipe, num processo permanente de prática e aprendizagem reflexiva. Assim, pode-se dizer que a perspectiva da relação recíproca entre educação e trabalho e a da abordagem pedagógica apoiada em metodologias ativas e problematizadoras de aprendizagem estão presentes tanto nos movimentos de EPS quanto nos de EIP.

Outro aspecto que se agrega à EPS é referente ao "quadrilátero da formação" que articula gestores, trabalhadores, instituições formadoras, docentes, estudantes, usuários e movimentos sociais envolvidos e comprometidos com essa construção em rede ensino-aprendizagem no SUS, na interação/atenção, gestão, ensino e controle social ${ }^{(3)}$. Essa ideia traz como pano de fundo que todos governam no cotidiano e disputam a direção da ação em saúde, fazendo uso dos recursos de que dispõem.

$\mathrm{Na}$ configuração teórico-conceitual da EPS e da EIP, encontram-se referenciais comuns quanto à aproximação teoria e prática, em particular no reconhecimento da relação intrínseca entre atenção à saúde e formação dos profissionais de saúde, isto é, o sistema de saúde e o sistema de educação são interdependentes e, com isso, impactam não apenas as práticas mas também as teorias ${ }^{(15)}$. A relação recíproca entre cuidados à saúde e formação profissional tanto reproduzem modelos de práticas e valores dominantes como constituem novos modelos e valores radicais orientados à mudança das práticas vigentes. Os quadros teóricos que expõem o profundo imbricamento entre a saúde e a formação são encontrados em diversas correntes, destacando-se as vertentes da pedagogia crítica e do construtivismo. Contudo, as relações estendidas pelo "quadrilátero da formação"(3) no tocante à gestão e ao controle social do SUS não estão presentes nas formulações da EIP, mesmo consideradas as peculiaridades dos sistemas de saúde de cada país, o reconhecimento de usuários, famílias e comunidade como partícipes das equipes e das práticas interprofissionais colaborativas e a tradição de participação social de alguns países como França, Reino Unido e outros.

A EPS se fundamenta em conceitos advindos da vertente histórico-estrutural e dialética como trabalho e processo de trabalho em saúde e concepção de saúde, doença e cuidado do campo da Saúde Coletiva. Nesse contexto, a saúde é resultante de condições de vida e, assim, as ações e práticas do setor saúde estão entrelaçadas ao modo de produção capitalista, às desigualdades sociais e aos interesses econômicos. A saúde e a educação são tomadas como práticas políticas no sentido de práticas com intencionalidades. Propõem interrogar a naturalização da diferença histórica de poderes entre gestores e trabalhadores, trabalhadores e trabalhadores e entre trabalhadores e usuários. Há ainda conceituações 
que vêm de autores do movimento institucionalista, como a noção de coletivos em análise, questionamento do saber dos especialistas sobre as pessoas, desvalorizando seus saberes e hierarquizando as relações. Articulam-se aos movimentos de educação popular em saúde e de problematização freiriana para que os coletivos reflitam sobre seus problemas e modos de funcionamento, produzam conhecimentos e se reconheçam produtores de si e do mundo.

A EIP preconiza o lema aprender juntos para trabalhar juntos ${ }^{(15)} \mathrm{e}$, com essa finalidade, as experiências em todo o mundo se orientam por correntes teóricas, transitando da cultura de iniciativas intuitivas ou pragmáticas para uma abordagem assentada em pressupostos teóricos e conceituais capazes de orientar o processo e o resultado das iniciativas. A adoção da EIP pode se orientar por várias teorias, mas algumas aparecem com mais frequência na literatura, evidenciando suas contribuições para o desenvolvimento de competências colaborativas. Dentre as mais citadas, tem-se: a teoria da aprendizagem de adultos de Clark, Schon, Dewey e Kolb adaptada às relações interprofissionais; teorias da educação e da psicologia voltadas aos processos de aprendizagem e teorias críticas da sociologia voltadas à análise dos desafios para interprofissionalidade; a psicodinâmica social para análise das interações e barreiras entre pessoas e grupos; a teoria do contato, que contribui para a compreensão dos estereótipos profissionais na perspectiva de Carpenter; teorias das identidades sociais, profissionais, relacionais e voltadas aos conflitos, nas quais se destaca o campo da sociologia das profissões; a teoria da prática de Pierre Bordieu; e a aprendizagem situada, baseada no princípio da comunidade de práticas, ou seja, práticas compartilhadas de aprendizagem ${ }^{(30)}$.

\section{CONSIDERAÇÕES FINAIS}

A valorização dos diferentes saberes profissionais e da participação dos usuários é fundamental tanto na EIP quanto na EPS, pois a principal finalidade desses movimentos está no compromisso com a atenção às necessidades de saúde dos usuários, famílias e comunidade, na perspectiva da integralidade do cuidado no SUS.

Destaca-se um aspecto reconhecido na análise teórico-conceitual e metodológica apresentada como inerente à EPS e a EIP. Ambas constituem disparadores de processos de mudança do trabalho e da formação dos profissionais de saúde pela não reprodução de assujeitados que o modelo hegemônico de cuidado e de educação produz para o lugar de sujeitos que se desconfortam com as formas de produzir saúde-doença no cotidiano (seja como trabalhadores, estudantes, gestores, usuários, famílias e comunidade), de modo que os participantes se abram para novos caminhos e possibilidades, colocando-se em permanente reflexão e produção.

É fundamental que os conceitos de EPS e EIP sejam compreendidos na sua natureza conceitual para que não percam sua potência, mas fortaleçam o processo de mudança das práticas em saúde no SUS. A EPS e a EIP possuem como diretriz comum a mudança da educação e formação de profissionais, como contribuição para a construção da atenção em saúde que produza cuidados e reconhecimento dos partícipes dos processos: trabalhadores, gestores, usuários, famílias e comunidade.

A EPS e EIP possuem histórias que se entrecruzam no Brasil e partem de bases teóricas conceituais diferentes. A EPS se baseia na vertente histórico-estrutural e dialética e do movimento institucionalista. A EIP se fundamenta em teorias advindas dos campos da educação de adultos, da psicodinâmica social, da sociologia das profissões e sociologia organizacional, entre outras. A EPS se propõe a trabalhar com o quadrilátero envolvendo formadores, gestores, trabalhadores e controle social, e a EIP pressupõe a interdependência entre formação profissional e atenção à saúde, com estímulo ao aprendizado interativo e compartilhado entre estudantes e trabalhadores de diferentes áreas para fomentar práticas colaborativas.

A implementação de ambas desafia, pois envolvem rupturas nas clássicas formas de organização e funcionamento das instituições formadoras de profissionais de saúde, dos serviços de saúde e das entidades de classe representantes das categorias profissionais.

É importante reafirmar que a EPS e a EIP são apostas para mudanças das práticas de saúde e de educação que têm raízes profundas e que, nesse sentido, são apostas políticas, cuja implementação requer a mobilização social de todos os atores envolvidos e a superação da disputa em interjogos de saberes e poderes, colocando como centro desses debates a construção de um sistema de saúde efetivamente integral e resolutivo.

\section{RESUMO}

Ensaio teórico de cunho reflexivo elaborado com base na literatura nacional e internacional, com o objetivo de analisar as interfaces e distinções histórico-conceituais entre Educação Permanente em Saúde e Educação Interprofissional em Saúde. No cenário internacional, ocorreram movimentos educacionais voltados para trabalhadores de saúde com destaque para os estímulos da Organização PanAmericana de Saúde. No Brasil, ambas as propostas ressaltam o compromisso com a qualidade das práticas no Sistema Único de Saúde, centradas nas necessidades de saúde com enfoques que se aproximam. A Educação Permanente em Saúde, comprometida com a formação no trabalho para a transformação do processo de trabalho, tem em vista o cuidado integral e a Educação Interprofissional em Saúde com o aprendizado interativo compartilhado de competências colaborativas para o efetivo trabalho em equipe, orientado pela colaboração interprofissional. Ambas visam à qualificação das práticas de saúde por meio da educação de trabalhadores em coletivos, mas é fundamental a distinção dos referenciais teórico-conceituais e metodológicos que as sustentam.

\section{DESCRITORES}

Educação em Saúde; Engajamento no Trabalho; Relações Interprofissionais; Educação Interprofissional.

\section{RESUMEN}

Ensayo teórico de corte reflexivo elaborado con base en la literatura nacional e internacional, objetivando analizar las interfaces y distinciones histórico-conceptuales entre Educación Permanente en Salud y Educación Interprofesional en Salud. En el ámbito 
internacional hubo movimientos educativos orientados a trabajadores de salud destacándose los estímulos de la Organización Panamericana de Salud. En Brasil, ambas propuestas subrayan el compromiso con la calidad de las prácticas del Sistema Único de Salud, centradas en necesidades sanitarias con enfoques cercanos. La Educación Permanente en Salud, comprometida con la formación laboral para transformar el proceso de trabajo considerando la atención integral, y la Educación Interprofesional en Salud con aprendizaje interactivo compartido de competencias colaborativas para un trabajo en equipo efectivo orientado por la colaboración interprofesional. Ambas apuntan a calificar las prácticas de salud educando al trabajador en colectivos, aunque resulta fundamental diferenciar los referenciales teóricos, conceptuales y metodológicos que las sustentan.

\section{DESCRIPTORES}

Educación en Salud; Compromiso Laboral; Relaciones Interprofesionales; Educación Interprofesional.

\section{REFERÊNCIAS}

1. Ferreira L, Barbosa JSA, Esposti CDD, Cruz MM. Permanent Health Education in primary care: an integrative review of literature. Saúde Debate. 2019;43(120):223-9. https://doi.org/10.1590/0103-1104201912017

2. Gadotti, M. A educação contra a educação: o esquecimento da educação e a educação Permanente. 5th ed. Rio de Janeiro: Paz e Terra; 1992.

3. Ceccim RB, Feuerwerker LM. A four-way approach to training in the health field: teaching, management, care, and social control. Physis. 2004;14(1):41-65. https://doi.org/10.1590/S0103-73312004000100004

4. Ceccim RB. [Permanent Education in the Healthcare field: an ambitious and necessary challenge]. Interface (Botucatu). 2005;9(16):161-8. Portuguese. https://doi.org/10.1590/S1414-32832005000100013

5. Peduzzi M, Agreli HLF, Silva JAM, Souza HS. [Teamwork: revisiting the concept and its developments in inter-professional work]. Trab Educ Saúde. 2020;18(suppl 1):e0024678. Portuguese. https://doi.org/10.1590/1981-7746-sol00246

6. Ramos M. Trabalho, educação e correntes pedagógicas no Brasil: um estudo a partir da formação dos trabalhadores técnicos da saúde. Rio de Janeiro: EPSJV/UFRJ; 2010.

7. Brasil. Ministério da Saúde. Portaria nº 198/GM/MS, de 13 de fevereiro de 2004. Política Nacional de Educação Permanente em Saúde. Brasília, DF: Ministério da Saúde; 2004

8. Reeves S, Fletcher S, Barr H, Birch I, Boet S, Davies N, et al. A BEME systematic review of the effects of interprofessional education: BEME Guide $n^{\circ}$ 39. Med Teach. 2016;38(7):656-68. https://doi.org/10.3109/0142159X.2016.1173663

9. Cavalcanti FOL, Guizardi FL. [Continued or permanent education in health? analysis of the production of the pan american health organization]. Trab Educ Saúde. 2018;16(1):99-122. Portuguese. https://doi.org/10.1590/1981-7746-sol00119

10. Brasil. Senado Federal. Constituição da República Federativa do Brasil. Brasília, DF: Senado; 1988.

11. Brasil. Ministério da Saúde. Secretaria de Gestão do Trabalho e da Educação na Saúde. Política Nacional de Educação Permanente em Saúde. Brasília, DF: Ministério da Saúde; 2009.

12. Gonçalves CB, Pinto ICM, França T, Teixeira CF. The resumption of the implementation process of the National Permanent Health Education Policy in Brazil. Saúde Debate. 2019;43(n spe 1):12-23. https://doi.org/10.1590/0103-11042019s101

13. Giovanella L, Franco CM, Almeida PF. National Primary Health Care Policy: where are we headed to?. Cienc Saúde Coletiva. 2020;25(4):1475-82. https://doi.org/10.1590/1413-81232020254.01842020

14. Declaração de Alma-Ata. Conferência Internacional sobre Cuidados Primários de Saúde; Alma-Ata, USSR, 6-12 de setembro de 1978. In: BRASIL. Ministério da Saúde. Secretaria de Políticas de Saúde. Projeto Promoção da Saúde. As cartas da promoção da saúde. Brasília, DF: Ministério da Saúde; 2002 [cited 2021 Apr 29]. p. 33-34 Available from: http://bvsms.saude.gov.br/bvs/publicacoes/cartas_promocao.pdf

15. World Health Organization. Learning together to work together for health. Report of a WHO Study Group on Multiprofessional Education for Health Personnel. Geneva: World Health Organization; 1988 [cited 2020 Sept 21]. (Technical Report Series, v. 769). Available from: https://apps.who.int/iris/bitstream/handle/10665/37411/WHO_TRS_769.pdf?sequence=1\&isAllowed=y

16. Frenk J, Chen L, Bhutta ZA, Crisp N, Evans T, Fineberg H, et al. Health professionals for a new century: transforming education to strengthen health systems in independent world. Lancet. 2010;376(9721):1923-58. https://doi.org/10.1016/S0140-6736(10)61854-5

17. World Health Organization. Framework for action on interprofessional education \& collaborative practice. Geneva: World Health Organization; 2010. [cited 2020 Sep 21]. Available from: https://apps.who.int/iris/bitstream/handle/10665/70185/WHO_HRH_HPN_10.3_eng.pdf?sequence=1

18. World Health Organization. Global Strategy on human resources for health: workforce 2030. Geneva: World Health Organization; 2016 [cited 2020 Sept 19]. https://doi.org/10.3109/13561829809014104

19. Barr H. Competent to collaborate: towards a competency-based model for interprofessional education. J Interprof Care. 1998;12(2):181-7. https://doi.org/10.3109/13561829809014104

20. Canadian Interprofessional Health Collaborative. Interprofessional education \& core competences: literature review [Internet]. Vancouver: University of British Columbia; 2007 [cited 2020 Sept 19]. Available from: https://pdf4pro.com/view/interprofessional-education-ampcore-competencies-cihc-4c6330.html

21. Horder J. A history of the Centre for the Advancement of Interprofessional Education (CAIPE) 1987 - 2003. London: CAIPE; 2003 [cited 2020 Sep 19]. Available from: https://www.caipe.org/resources/publications/archived-publications/history-centre-advancementinterprofessional-education-caipe-1987-2003-john-horder-first-chairman

22. Teixeira CFS, Ávila DCMT, Nunes DRM. [Interdisciplinary Bachelor's degree in Health: an innovative proposal in higher education in Health in Brazil]. Cienc Saúde Coletiva. 2013;18(6):1635-46. Portuguese. https://doi.org/10.1590/S1413-81232013000600015

23. Veras RM, Coelho MTAD, Teixeira CM, Traverso-Yepez MA. [The cycle system training in the Interdisciplinary Bachelor of Health at the Universidade Federal da Bahia and the Interprofessional Education proposal]. Avaliação (Campinas). 2018;23(2):294-311. Portuguese. https://doi.org/10.1590/s1414-40772018000200002 
24. Câmara AMCS, Cyrino AP, Cyrino EG, Azevedo GD, Costa MV, Bellini MIB et al. [Interprofessional education in Brazil: building synergic networks of educational and healthcare processes]. Interface (Botucatu). 2016;20(56):5-8. Portuguese. https://doi. org/10.1590/1807-57622015.0700

25. World Health Organization; Pan American Health Organization. Regional Network for Interprofessional Education in the Americas [Internet]. Geneva: PAHO; 2017 [cited 2020 Sept 20]. Available from: https://www.educacioninterprofesional.org/en

26. Brasil. Ministério da Saúde. Secretaria de Gestão do Trabalho e da Educação na Saúde. Departamento de Gestão da Educação na Saúde. Relatório final da oficina de alinhamento conceitual sobre educação e trabalho interprofissional em saúde [Internet]. Brasília, DF: Ministério da Saúde; 2017 [cited 2021 Apr 29]. Available from: https://www.educacioninterprofesional.org/sites/default/files/fulltext/2018/ pub_relatoria_eip_bra_2017_po.pdf

27. Merhy EE, feuerwerker LCM, Ceccim RB. [Permanent education in health: a strategy for acting upon the micropolitics of the work in health]. Salud Colectiva [Internet]. 2006 [cited 2018 dez. 19]:2(2):147-160. Spanisch. Available from: http://www.scielo.org.ar/scielo. php?script=sci_arttext\&pid=S1851-82652006000200004\&lng=es

28. Costa MV, Freire Filho JR, Brandão C, Silva JAM. Education and interprofessional practice in line with the historical commitment to strengthen and consolidate the Brazilian National Health System (SUS). Interface (Botucatu). 2018;22(suppl 2):1507-10. https://doi. org/10.1590/1807-57622018.0636

29. Shrader s, Jernigan S, Sick B. Developing interprofessional preceptors to promote intentional interprofessional education in practice settings: reflections from the preceptors in the Nexus Workshop. JCIPE [Internet]. 2020 [cited 2020 Sept 24];10(2):4. Available from: https://jdc. jefferson.edu/jcipe/vol10/iss2/4

30. Barr H. Toward a theoretical framework for interprofessional education. J Interprof Care. 2013;27(1):4-9. https://doi.org/10.3109/13561 820.2012 .698328 